\title{
NOD2-expressing bone marrow-derived cells appear to regulate epithelial innate immunity of the transplanted human small intestine
}

\author{
T Fishbein, ${ }^{1,2,6}$ G Novitskiy, ${ }^{1,2}$ L Mishra, ${ }^{3,4}$ C Matsumoto, ${ }^{1,2}$ S Kaufman, ${ }^{1,2,6}$ S Goyal, ${ }^{2}$ \\ K Shetty, ${ }^{1,2,6} \mathrm{~L}$ Johnson, ${ }^{1,2} \mathrm{~A} \mathrm{Lu},{ }^{1,2} \mathrm{~A}$ Wang, ${ }^{7} \mathrm{~F} \mathrm{Hu},{ }^{7}$ B Kallakury, ${ }^{5} \mathrm{D}$ Lough, ${ }^{2} \mathrm{M}$ Zasloff ${ }^{1,2,6}$
}

${ }^{1}$ Transplant Institute, Georgetown University Hospital, Washington, DC, USA; ${ }^{2}$ Department of Surgery, Georgetown University Medical Center, Washington, DC, USA;

${ }^{3}$ Laboratory of GI Developmental Biology, Departments of

Surgery, Medicine and The Lombardi Comprehensive Cancer Center, Georgetown University, Washington, DC, USA

${ }^{4}$ DVAMC, Washington, DC USA; ${ }^{5}$ Department of Pathology, Georgetown University Medical Center, Washington, DC, USA;

${ }^{6}$ Department of Pediatrics, Georgetown University Medical Center, Washington, DC, USA; ${ }^{7}$ Department of Biostatistics, Lombardi Comprehensive Cancer Center, Georgetown University, Washington, DC, USA

Correspondence to:

Dr Michael Zasloff, Georgetown University Medical Center, Med/ Dent Bldg, NW 210, 3900 Reservoir Rd, NW, Washington, DC 20007, USA; maz5@ georgetown.edu

Revised 10 October 2007 Accepted 12 October 2007 Published Online First 19 October 2007

\section{ABSTRACT}

Background: Intestinal allograft rejection resembles Crohn's disease clinically and pathologically. An understanding of its mechanism could impact this life-saving procedure, as well as provide insight into the pathophysiology of inflammatory bowel disease. The NOD2 protein has been implicated as a key player in intestinal immune health, as a consequence of the discovery of three polymorphisms linked with Crohn's disease. An investigation was carried out to determine whether epithelial immune function and graft survival were influenced by NOD2 mutations in an intestinal transplant population.

Methods: The NOD2 genotypes of 34 transplants performed consecutively over the past 3 years were determined. The NOD2 genotypes were related to clinical outcomes and the expression of certain intestinal antimicrobial peptides (AMPs) believed to protect the epithelium.

Results: An unexpectedly high percentage of recipients, $35 \%$, possessed NOD2 polymorphisms, while $8.6 \%$ of donors had comparable mutations. The likelihood of allograft failure was about 100-fold higher in recipients with mutant NOD2 alleles compared with recipients with wild-type NOD2 loci. Rejection in NOD2 mutant recipients was characterised by decreased expression of certain Paneth cell and enterocyte AMPs, prior to the onset of epithelial injury and inflammation.

Conclusions: Crohn's disease-associated polymorphisms in the NOD2 gene in the recipient represent a critical immunological risk factor for intestinal allograft rejection. Compromised epithelial defences precede visible epithelial injury and inflammatory infiltration. The association of impaired epithelial immunity with the recipient's genotype suggests that certain NOD2-expressing cells of haematopoietic origin play a role in the process, perhaps by regulating expression of certain epithelial AMPs within the allograft.

The NOD2 gene product has recently been recognised to play an important role in the immune health of the small intestine. The discovery arose from efforts to identify genes that were associated with Crohn's disease (CD), a disorder in which intestinal inflammation is provoked by commensal bacteria. NOD2 is an intracellular microbial sensor present in macrophages, dendritic cells and certain intestinal epithelial cells, including Paneth cells. ${ }^{1-3}$ Several alleles in the NOD2 locus have been identified as potentially functionally abnormal, and the risk of having CD increases between 2- and 40-fold depending on the genotype of the individual. ${ }^{4-8}$
Studies in both mouse and man have linked the presence of NOD2 CD-associated polymorphisms with impaired expression of certain Paneth cellderived antimicrobial peptides (AMPs) ${ }^{9}{ }^{10}$ in individuals with the ileal form of $\mathrm{CD} .{ }^{11-13}$ The hypothesis proposes that the presence of impaired NOD2 sensors results in the failure to regulate intestinal epithelial AMPs effectively; subsequent epithelial damage caused by commensal microbes results in a secondary tissue-destructive inflammatory response.

More than 600 people worldwide have received a small intestinal allograft over the past 15 years for intestinal failure following surgical resection or functional intestinal anomalies. ${ }^{14-18}$ About $60 \%$ of the recipients are infants and children younger than 15 years for whom the allograft provides the only remaining means to deliver adequate nutritional support required for growth. ${ }^{14} 1819$ Graft failure secondary to rejection occurs in about 30 $40 \%$ of transplants within the first 3 years postoperatively. Efforts continue to define optimal strategies to increase survival of the allograft.

Small intestinal allograft rejection is characterised by intestinal crypt cell damage and apoptosis, invasion of the lamina propria by lymphocytes and macrophages, and, in later stages, luminal epithelial ulceration or complete destruction, reminiscent of some of the pathological characteristics of both CD and graft versus host disease. ${ }^{20} 21$

These reports prompted us to examine whether an association existed between the presence of NOD2 CD-linked polymorphisms in our transplant population and transplant outcome. We hypothesised that the presence of a NOD2 polymorphism in the recipient might influence the viability of the allograft by interrupting NOD2dependent circuits required to maintain intestinal homeostasis with respect to commensal flora. Outcomes that were selected for assessment included the first episode of severe rejection and time to graft failure post-transplantation. Furthermore, we speculated that damage to the intestinal epithelium of a healthy bowel (recognised as "rejection") would be more likely to occur in a recipient lacking a functional intestinal microbial-sensing system than in a recipient with an intact system, and that the damage would be more severe pathologically.

We further questioned whether the presence of a NOD2 polymorphism was associated with alteration in the expression of Paneth cell and enterocyte AMP genes of the allograft after implantation, a
This paper is freely availat online under the BMJ Journa unlocked scheme, see http:/ gut.bmj.com/info/unlocked.dt 
deficiency in epithelial innate immunity observed in CD.

\section{METHODS \\ Study population}

Between May 2002, the date when the intestinal transplant programme began, and November 2006, 34 consecutive patients underwent small intestinal transplantation at the Georgetown University Hospital under the direction of a single surgeon (TF). The study population includes every individual who received an intestinal allograft at our institution, with the last patient included having about 4 months postsurgical time. DNA samples suitable for genotyping, tissue specimens, along with complete clinical information from recipients and donor organs were obtained with approval of the Georgetown University Institutional Review Board. The authors were responsible for the design of the study, assembly and analysis of the data, and the writing of the manuscript.

The study described in this report began in mid-2005 after about 20 patients had been transplanted. The transplantation protocol and all postoperative management procedures were not altered by the initiation of this study and thus remained fixed for the entire group of 34 patients. All individuals involved in patient management were unaware of the NOD2 genotypes of the recipients and donors.

The following clinical data were collected on all recipients: age, sex, race, serological evidence of cytomegalovirus (CMV) or Epstein-Barr virus (EBV) infection, the primary cause of intestinal failure, organs transplanted in addition to the small intestine, the duration of cold ischaemia, the number of episodes of rejection, the histological severity of rejection, the number of episodes of infection and responsible organisms, the loss of the graft, and vital status, with the cause of death established. Donor information included age, sex, race, CMV and EBV status. Donor organs were all from heart-beating cadaveric donors who suffered irreversible brain injury but who had normal bowel function and no history of gastrointestinal disease. Recipients were individuals who suffered intestinal failure and lifelong dependence on parenteral nutrition. One patient was retransplanted after the loss of the first allograft. The majority had short bowel syndrome, having required massive surgical resection of the small intestine due to a variety of common paediatric and adult diseases. Other patients suffered either functional disorders of motility or had desmoid tumours associated with familial adenomatous polyposis (table 1). Our population did not differ with respect to the reasons for transplantation from the experience of other centres. ${ }^{15} 172223$

The basic transplantation protocol has been described in detail elsewhere. ${ }^{14} 1819$ Rejection was routinely treated with pulsed steroid and augmented tacrolimus therapy when mild, and with antilymphocyte agents when more severe, as clinically indicated. Bacterial decontamination of the intestine was performed when signs of systemic toxaemia accompanied rejection. Endoscopic biopsy evaluations and symptom evaluation guided daily management decisions.

\section{Evaluation of allograft rejection}

Endoscopic mucosal pinch biopsies were taken at transplant protocol-specified time points and additionally whenever clinically indicated. Patients consented to additional biopsy material being collected simultaneously for the purpose of evaluation of the expression of AMPs. Biopsy samples were fixed, paraffin embedded and stained with H\&E. Biopsies were then examined by one of two pathologists unaware of the NOD2 status of the patient and scored using standard rejection grading criteria. ${ }^{15} 24$

Amongst the earliest microscopic signs of acute small bowel rejection are apoptotic cells within the crypts, increasing in number/crypt as rejection progresses (fig 1A,B). As the severity of rejection increases, villous blunting is seen, followed by invasion of the lamina propria and crypt of inflammatory cells, with eventual mucosal erosion (fig 1C-F). "Severe rejection" as referred to in this report describes pathology characterised by extensive mucosal ulceration and inflammatory infiltration of the lamina propria. Episodes of rejection separated by $>30$ days with an intervening period characterised by normal histology were considered as independent events.

\section{Tissue sample collection}

Grasp biopsies were obtained from allograft mucosa during either endoscopy of the ileal stoma or colonoscopy following stoma takedown, generally between 10 and $30 \mathrm{~cm}$ from the ileocolonic anastomosis. In patients with ileal ulceration or other pathologies, biopsies were targeted to regions of intact mucosa. Samples of pre-reperfusion donor small bowels and biopsies were placed into RNALater (Ambion, Austin, TX) according to the manufacturer's instructions and saved at $-80^{\circ} \mathrm{C}$ until used.

\section{DNA extraction}

Isolation of genomic DNA from tissue (OIAamp DNA Mini Kit; Oiagen, Valencia, CA) or blood (OIAamp DNA Blood Mini Kit, Qiagen) was done according to the manufacturer's recommendations. EDTA-anticoagulated blood was spun for $10 \mathrm{~min}$ at $2000 \mathrm{rpm}$ at $4^{\circ} \mathrm{C}$; the buffy coat was collected and stored at $80^{\circ} \mathrm{C}$ until used for DNA isolation.

\section{Genotyping assay}

All donors and recipient were genotyped for three common single nucleotide polymorphisms (SNPs) in the NOD2/CARD15 gene: (1) a missense mutation 2104C $\rightarrow \mathrm{T}$ (SNP 8; R702W, NCBI reference SNP ID: rs2066844); (2) a missense mutation 2722 $\mathrm{G} \rightarrow \mathrm{C}$ (SNP 12; G908R, NCBI reference SNP ID: rs2066845); and (3) a frameshift C-mutation, 3020insC (SNP 13; 1007fs, NCBI reference SNP ID: rs2066847). For R702W and G908R mutation, two pre-made (Assay-on-Demand) assays were provided by Applied Biosystems (Assay ID: c_ _ 11717468_20 and c_ _ 11717466_20). For the 1007fs mutation, the assay was customdeveloped by Applied Biosystems with the following primers and fluorophor-labelled probes: (forward primer, GTCCAA TAACTGCATCACCTACCT; reverse primer, CAGACTT CCAGGATGGTGTCATTC; VIC-labelled probe, CAGGCC CCTTGAAAG; FAM-labelled probe, CAGGCCCTTGAAAG). Reactions were performed according to the manufacturer's instructions in a 96-well plate. Briefly, 10 ng of genomic DNA was mixed with $12.5 \mu \mathrm{l}$ of $2 \times$ TaqMan Universal PCR Master Mix No AmpErase UNG and $1.25 \mu \mathrm{l}$ of $20 \times$ SNP Genotyping Assay, and PCR was carried out on a 7500 Fast Real Time PCR instrument (Applied Biosystems, Foster City, CA). Thermal cycling conditions were: $95^{\circ} \mathrm{C}$ for $10 \mathrm{~min}$ followed by 40 cycles of $92^{\circ} \mathrm{C}$ for $15 \mathrm{~s}$ (melting step) and $60^{\circ} \mathrm{C}$ for $60 \mathrm{~s}$ (anneal/extend step). Detection of fluorescent signal was performed according to the recommended protocols for the 7500 Fast Real Time PCR machine, and the results were analysed by the associated Sequence Detection System (SDS) Software V. 1.3.0. (Applied Biosystems). 
Figure 1 Pathological characteristics of small intestinal allograft rejection. (A) Crypt apoptosis; (B) multiple apoptotic bodies; (C) villous blunting; (D) inflammatory cell infiltration; (E) cryptitis; (F) mucosal ulceration (H\&E).
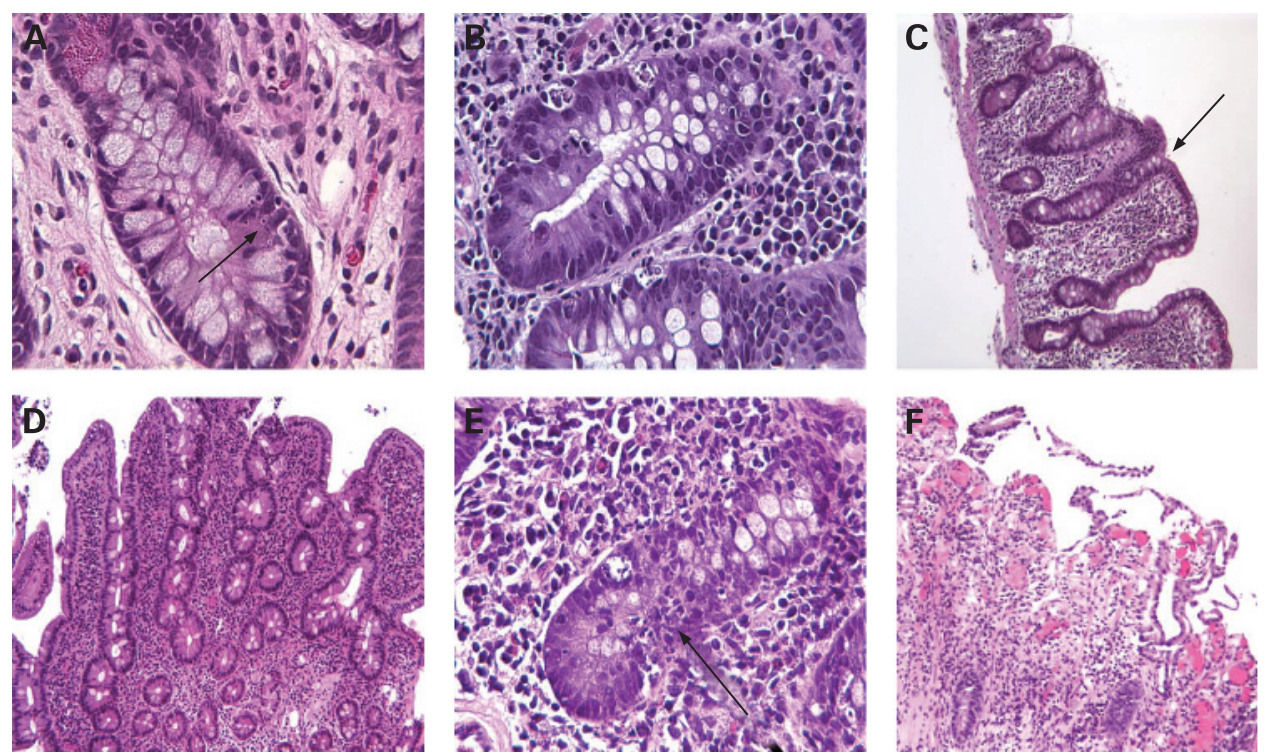

\section{Determination of mRNA abundance by TaqMan quantitative reverse transcriptase-PCR (TaqMan qRT-PCR)}

The gene products analysed included antimicrobial peptides/ proteins expressed by Paneth cells (HD5, HD6 and lysozyme) and enterocytes (HBD1, HBD2, HBD3 and LL37). All donor organs examined were from individuals with normal NOD2 alleles.

Isolation of total RNA was performed by RNeasy Mini RNA isolation kit (Qiagen) according to the manufacturer's recommendations, from tissues that had been previously stored in RNALater (see above). RNA was quantified with a Biorad spectrophotometer; $A_{260 / 280}$ readings between 1.8 and 2.0 were used to confirm purity. Additionally, formaldehyde gel electrophoresis was performed to verify the presence of intact $18 \mathrm{~S}$ and $28 \mathrm{~S}$ rRNA. The expression of human AMP mRNA in small bowel was determined by TaqMan qRT-PCR. To measure cDNA levels, the threshold cycle at which fluorescence is first detected above baseline is used, and a standard curve is drawn between starting nucleic acid concentrations and the threshold cycle.

We measured the concentration of the following AMP mRNAs: human $\beta$ defensin 1 (hBD1, NCBI RefSeq: NM 005218.3), human $\beta$ defensin 2 (hBD2, NCBI RefSeq: NM_004942.2), human $\beta$ defensin 3 (hBD3; NCBI RefSeq: NM_018661.2), human defensin 5 (HD5, NCBI RefSeq: NM 021010.1), human defensin 6 (HD6, NCBI RefSeq: NM_001926.2), lysozyme (Lsz, NCBI RefSeq: NM_021797.2) and cathelicidin-derived peptide LL37 (LL37, NCBI RefSeq: NM_004345.3). Pre-made TaqMan probes and primers (assay ID: $\mathrm{Hs} 00608345 \mathrm{~m} 1, \quad \mathrm{Hs} 00175474 \mathrm{~m} 1, \quad \mathrm{Hs} 00218678 \mathrm{~m} 1$, Hs00360716_m1， Hs00427001_m1, Hs00253976_m1, Hs00189038_m1, respectively) were purchased from Applied Biosystems. Target mRNA levels are expressed relative to an internal glyceraldehyde-3-phosphate dehydrogenase (GAPDH) control (assay ID: Hs99999905_m1, Applied Biosystems). Isolated total RNA $(1 \mu \mathrm{g})$ was reverse-transcribed into cDNA in a $20 \mu$ reaction mix using Superscript III reverse transcriptase and random hexamers, according to the manufacturer's instructions (Invitrogen, Carlsbad, CA). For cDNA amplification, a 10 min incubation at $95^{\circ} \mathrm{C}$ was carried out to activate AmpliTaqGold DNA polymerase; this was followed by 40 cycles with $15 \mathrm{~s}$ at $95^{\circ} \mathrm{C}$ and $1 \mathrm{~min}$ at $60^{\circ} \mathrm{C}$ for each cycle.
The relative expression was defined as a relative value in comparison with the arbitrary expression value 1 obtained from patients without any polymorphism in NOD2/CARD15 or as qualified in fig 3. qRT-PCR was performed with the 7500 Fast Real Time PCR machine (Applied Biosystems). More information can be obtained from https://products.appliedbiosystems.com/ ab/en/US/adirect/custom_taqman_assay_link_placeholder.html

\section{Western blot analysis}

Ileal tissue (5-8 mg) was analysed by acid urea-polyacrylamide gel electrophoresis (AU-PAGE), followed by western blotting as described ${ }^{26}$ The tissue was homogenised in ice-cold acetic acid $(1: 10 \mathrm{w} / \mathrm{v})$, containing $1 \mathrm{mM}$ phenylmethylsulphonyl fluoride, $1 \mu \mathrm{g} / \mathrm{ml}$ leupeptin and $1 \mu \mathrm{g} / \mathrm{ml}$ aprotinin. Samples $(25 \mu \mathrm{g} /$ line $)$ were subjected to $12.5 \%$ AU-PAGE, transferred onto a polyvinylidene difluoride (PVDF) membrane, fixed with $0.1 \%$ glutaraldehyde in Tris-buffered saline (TBS) and blocked for $1 \mathrm{~h}$ in $5 \%$ non-fat powdered milk in TBS containing $0.1 \%$ Tween- 20 (TTBS). The membranes were then incubated overnight with the primary antibodies against hBD1, hBD2, HD5 and HD6 at a final dilution of 1:1000. Blots were washed several times in TTBS, and peroxidase-conjugated secondary antibodies were added for $2 \mathrm{~h}$. All antibodies were purchased from Alpha Diagnostic International Inc. (San Antonio, TX). Finally, membranes were washed several times in TTBS, and complexes were detected using the enhanced chemiluminescence detection (ECL) method (Amersham Biosciences, Piscataway, NJ). Protein levels were measured by Bradford assay. Relative densitometry of the bands was quantified using National Institute of Health ImageJ software, version 1.36B. $\beta$-Actin was used to confirm the load of protein in each lane.

\section{Paneth cell histology}

Tisssues were stained with phloxine-tartrazine as described ${ }^{13} \mathrm{At}$ least 10 crypts displaying Paneth cells were examined per patient, from 2-3 biopsies taken from physically separate sites on the distal ileum.

\section{Statistical analysis}

The major outcome variables (first episode of severe rejection, graft loss and patient death) were analysed in relation to NOD2 
Table 1 Recipient donor demographic and clinical characteristics grouped by NOD2 genotype

\begin{tabular}{|c|c|c|c|c|c|c|}
\hline \multirow[b]{2}{*}{ Characteristic } & \multicolumn{3}{|c|}{ NOD2 status-recipient } & \multicolumn{3}{|c|}{ NOD2 status—donor } \\
\hline & Wild-type $(n=22)$ & Mutant $(n=12)$ & $\mathrm{p}$ value & $\begin{array}{l}\text { Wild-type } \\
(n=32)\end{array}$ & $\begin{array}{l}\text { Mutant } \\
(\mathrm{n}=3)\end{array}$ & p value \\
\hline Sex & & & 1.00 & & & 0.21 \\
\hline Male & 13 & 7 & & 23 & 3 & \\
\hline Female & 9 & 5 & & 9 & 0 & \\
\hline Age & & & 0.65 & & & 0.71 \\
\hline$<10$ years & 12 & 7 & & 21 & 3 & \\
\hline $10-20$ years & 1 & 2 & & 3 & 0 & \\
\hline $20-40$ years & 3 & 1 & & 5 & 0 & \\
\hline$>40$ years & 6 & 2 & & 3 & 0 & \\
\hline Race & & & 0.94 & & & 0.33 \\
\hline Black & 7 & 5 & & 9 & 0 & \\
\hline White & 13 & 7 & & 18 & 3 & \\
\hline Other & 2 & 0 & & 5 & 0 & \\
\hline Primary pathology & & & 0.69 & & & \\
\hline Short gut syndrome & 16 & 7 & & & & \\
\hline Malrotation & 3 & 2 & & & & \\
\hline Gastroschesis & 5 & 2 & & & & \\
\hline Necrotising enterocolitis & 2 & 1 & & & & \\
\hline Jejunal atresia & 3 & 1 & & & & \\
\hline Gardner syndrome & 1 & 1 & & & & \\
\hline Hirschsprung & 1 & 0 & & & & \\
\hline Prune belly syndrome & 1 & 0 & & & & \\
\hline Crohn's disease & 1 & 2 & & & & \\
\hline Motility disorder & 3 & 2 & & & & \\
\hline Berdon syndrome & 0 & 2 & & & & \\
\hline Idiopathic pseudo-obstructior & 3 & 0 & & & & \\
\hline Tumour & 2 & 0 & & & & \\
\hline Other & 1 & 1 & & 32 & 3 & \\
\hline Trauma & 1 & 1 & & 32 & 3 & \\
\hline Allograft implanted & Wild-type $(\mathrm{n}=23)$ & Mutant $(n=12)$ & 0.96 & & & \\
\hline Small bowel & 9 & 5 & & & & \\
\hline Small bowel and liver & 8 & 4 & & & & \\
\hline Multivisceral* & 6 & 3 & & & & \\
\hline $\begin{array}{l}\text { Individual with any NOD2 } \\
\text { mutation }\end{array}$ & $12 / 34$ & & & $3 / 35$ & & 0.008 \\
\hline $\begin{array}{l}\text { Excluding individuals with } \\
\text { Crohn's diseas }\end{array}$ & $10 / 31$ & & & $3 / 35$ & & 0.014 \\
\hline
\end{tabular}

mutant alleles. Time to the clinical event was examined by means of Kaplan-Meier survival curves using log-rank analysis in order to determine significance. Cox's proportional hazards model was fit to control the possible effects from other factors. We compared the Kaplan-Meier curves of outcomes of NOD2 wild-type and NOD2 mutant recipients with a log rank test (fig 2), and then, separately fitting a Cox proportional hazard model to accommodate other potential influences on outcome (table 2). For patient demographic characteristics, a two-tailed unpaired t test was used for comparison of continuous variables, while Fisher's exact test was used for categorical variables or discrete variables. $p$ Values of $<0.05$ were considered to indicate statistical significance; all $p$ values were two-sided.

\section{RESULTS}

\section{Prevalence of NOD2 genotypes in recipients and donors}

NOD2 genotypes were determined for all 34 donor-recipient pairs. The median duration of follow-up for these patients was 1.3 years (range, 70 days to 4.5 years). Twelve of the 34 recipients $(35 \%)$ had one or more of the three NOD2 polymorphisms associated with CD (R720W, G908R,
3020insC), while 22 had corresponding wild-type NOD2 loci (table 1 ). In contrast, only $3 / 35$ donors (8.6\%) had CD NOD2 polymorphisms $(p<0.008)$. Each of the three NOD2 polymorphisms was represented in the recipient population, with no apparent bias towards a specific mutation. Several individuals carried two polymorphisms within a single NOD2 gene, and six individuals were homozygous for one of the CD NOD2 polymorphisms. A full description of the NOD2 hapolytypes observed in the transplant recipient population will be published elsewhere (Novitskiy et al, manuscript in preparation) Three recipients (8.8\%) had prior CD, two with NOD2 CDassociated polymorphisms and one without. To the best of our knowledge, none of the other recipients were first-degree relatives of individuals with $\mathrm{CD}$. The frequency of NOD2 polymorphisms in the donor group was within the range reported for the general population. ${ }^{4}$

\section{Association of NOD2 genotype with outcome}

Allograft recipients were divided into two groups according to whether or not the recipient carried a NOD2 CD-associated polymorphism (table 1). 
The median follow-up time was similar for the recipients with NOD2 polymorphisms and those with wild-type loci (1.4 and 1.2 years, respectively). Only three allografts were from donors with NOD2 polymorphisms, thus, our assessment of outcomes is essentially a reflection of the impact of the NOD2 genotype of the recipient on the transplant outcome. Two of the three allografts that came from donors with NOD2 CDassociated polymorphisms had been implanted into recipients who also had NOD2 CD alleles, including an individual with underlying $\mathrm{CD}$. Both experienced severe graft rejection, and the recipient with $\mathrm{CD}$ died of sepsis following allograft failure. The remaining NOD2 mutant donor graft was transplanted into a recipient with a wild-type NOD2 genotype and they experienced an uneventful course.

From the Kaplan-Meier curves we see that the risks of developing an initial severe episode of rejection (fig 2A) and of graft failure (fig $2 \mathrm{~B}$ ) were significantly greater in the NOD2 mutant recipients compared with the NOD2 wild-type recipients $(p=0.01$ and $p=0.002$, respectively). No patient deaths were observed in NOD2 wild-type recipients; each NOD2 mutant recipient who experienced graft rejection subsequently died prior to re-transplantation.

We considered as potential covariates the age, gender, prior viral status of the recipients and donors (CMV and EBV), and whether or not other organs were being transplanted along with the small intestine (table 2). The inclusion of the liver with the small intestine has been proposed to worsen outcome. ${ }^{18}$ Recipients with a NOD2 polymorphism were at greater risk of experiencing a first episode of severe, graft-threatening rejection than NOD2 wild-type recipients, with a hazard ratio of $4.449(\mathrm{p}<0.018)$; no significant effect was observed from other covariates in a Cox regression model (not shown). Similarly, the risk of graft loss was significantly greater for recipients with mutant NOD2 alleles than those with wild-type loci (hazard ratio 97.3, $p<0.006$ ). No deaths were found in the recipients with NOD2 wild-type alleles $(0 / 22)$, compared with $5 / 12(42 \%)$ of the NOD2 mutant group, representing a highly significant difference using Fisher's exact test $(p=0.0024)$.

\section{Association of recipient NOD2 genotype with graft AMP expression}

To assess whether the NOD2 genotype of the recipient influences the innate defences of the allograft, we examined the expression of epithelial AMPs in biopsy specimens obtained at a common time point (3-4 weeks) postoperatively from all recipients of wild-type allografts who had not yet experienced a rejection episode and who were free of gastrointestinal or systemic infections. Ten patients met these criteria, comprising four individuals with NOD2 polymorphisms and six with wildtype loci. Small bowel allografts implanted into both groups of recipients showed no significant difference in the overall expression of the AMP genes, as anticipated (fig 3A). Allografts introduced into wild-type recipients retained the same pretransplant level in the expression of these AMP genes 3-4 weeks after transplantation (fig 3B), despite active immunosuppression and prior surgery. In contrast, allografts implanted into the NOD2 mutant recipients exhibited significant depression of HD5 and HBD2 expression relative to pretransplant levels, despite normal bowel function and histology (fig 3C). The relative differences in mRNA content obtained by qPCR were confirmed by quantitative measurement of absolute mRNA copy numbers (Novitskiy et al, manuscript in preparation). Semi-quantitative analysis of protein extracted from the biopsy tissues was consistent with the relative differences in corresponding mRNA abundance (fig 3D-G). A light microscopic examination of biopsies taken from allografts at this time revealed no change in numbers of crypts or the number of Paneth cells per crypt. However, we observed a distinct decrease in size and number of phloxinetartrazine-stained secretory granules in recipients with NOD2 polymorphisms compared with wild-type recipients (fig $3 \mathrm{H}, \mathrm{I}$ ); Three of four recipients with NOD2 mutations selected for this analysis subsequently developed acute severe rejection between 2 and 6 months after transplantation, while none of the six wild-type recipients has as yet experienced severe rejection (between 1 and 2 years post-transplantation). Taken together, these data suggest that the "normal" expression of certain intestinal epithelial AMPs is not sustained when the intestine is introduced into a recipient with a mutant NOD2 genotype.

\section{DISCUSSION}

Our study demonstrates that small intestinal allograft recipients with one or more NOD2 CD-associated polymorphisms were more likely to suffer severe allograft rejection, allograft loss and death than recipients with corresponding wild-type loci. We suggest that NOD2 genotyping has important prognostic value in small intestinal transplantation.

NOD2 is an intracellular microbial sensor expressed most robustly in human macrophages, dendritic cells and Paneth cells. ${ }^{13}$ The importance of the NOD2 gene in human innate immunity was first appreciated by its identification as a locus linked to $\mathrm{CD},{ }^{5-8}$ but precisely how the defective NOD2 locus leads to $\mathrm{CD}$, as well as its role in intestinal health, remains controversial. Data from mouse and man support several hypotheses. Defects in the NOD2 gene of the intestinal macrophage have been shown to disturb the expression of the suite of cytokines necessary to co-ordinate the balance between immune suppression and activation required for healthy functioning of the adaptive immune system in the intestine; as a consequence, the presence of commensal microbes in individuals with $\mathrm{CD}$ is hypothesised to provoke a dysfunctional inflammatory reaction leading to epithelial injury. ${ }^{1}$ In addition, the NOD2 gene plays a role in maintaining the functional integrity of the healthy intestinal mucosal barrier; for example, individuals with both SNPs R702W and 3020insC are more likely to exhibit abnormal mucosal permeability than individuals with wild-type alleles. ${ }^{27}$ Other studies suggest that CDassociated defects in the NOD2 gene of the intestinal epithelial cell result in impaired expression of certain AMPs, leading to microbe-induced epithelial damage; a secondary inflammatory response is provoked to "protect" the intestinal mucosa. ${ }^{11} 1328$ Indeed, in a study performed with mice in which the NOD2 loci were genetically deleted, the expression of specific Paneth cell defensins (Defcr4, Defcr-rs10) was found to be significantly depressed compared with wild-type animals; the homozygotic knockout mice could not clear orally administered Listeria monocytogenes from their gastrointestinal tracts as efficiently as wild-type animals, although they handled a systemically administered inoculum indistinguishably. ${ }^{12}$

We have observed that epithelial AMP expression in the allograft is depressed in recipients with NOD2 CD loci prior to the appearance of histopathology visible by light microscopy. The AMPs involved, HD5 and HBD2, are both believed to protect the epithelium from microbial damage and control the growth and species diversity of commensals. These observations are consistent with those reported by Wehkamp et al in studies in ileal CD; depressed expression of Paneth cell HD5 was observed from biopsies taken both from areas of small intestine 

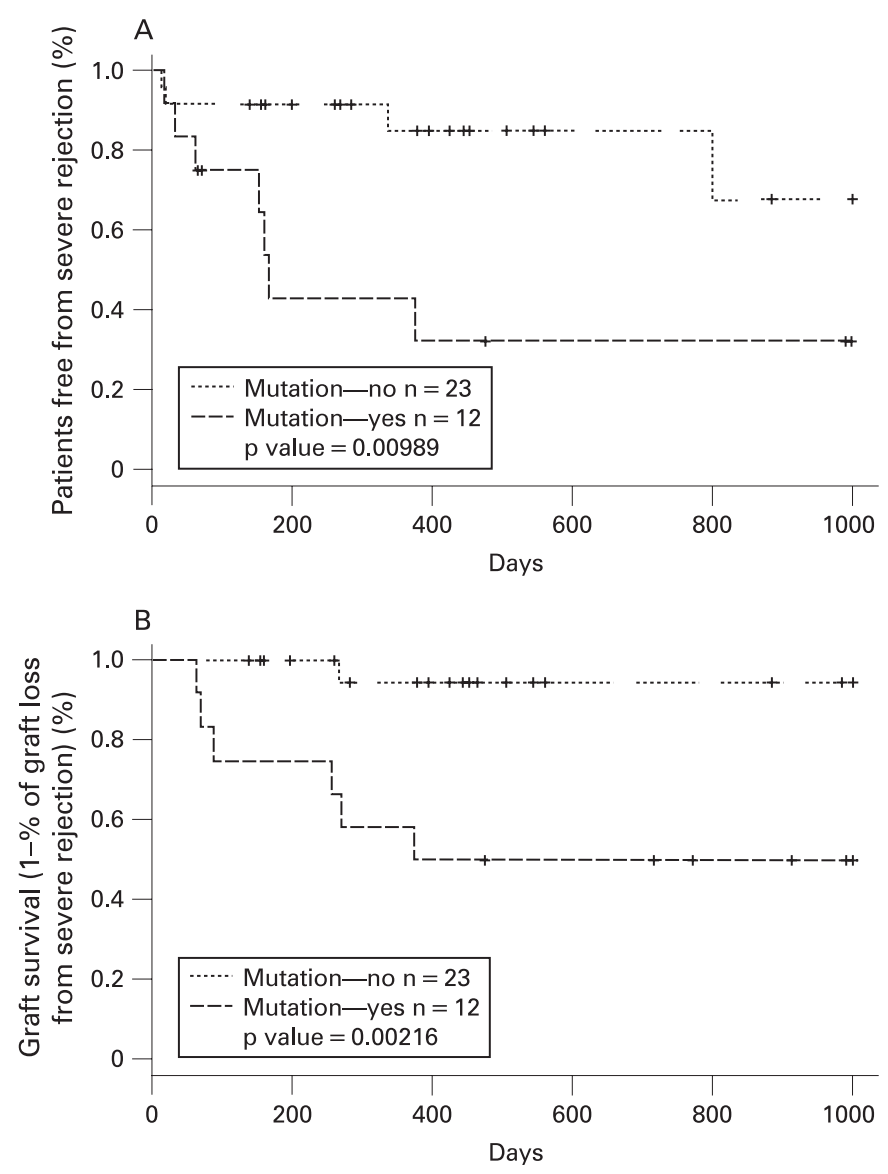

Figure 2 Kaplan-Meier curves of rejection and graft loss after transplantation associated with the recipient's NOD2 genotype. (A) Time to first episode of severe rejection; (B) time to loss of the allograft. One NOD2 wild-type recipient was transplanted twice and was scored as two transplants. Significance was determined by a log-rank test (see the Methods section).

that were inflamed and from those not pathologically diseased. ${ }^{13}{ }^{29}$ In addition, as observed here, Wehkamp et al noted that in ileal CD Paneth cell defensin expression was reduced while the numbers of Paneth cells per crypt were not. ${ }^{13}{ }^{29}$

It remains to be determined if this failure of AMP-based antimicrobial defences is directly responsible for the subsequent rejection process that more frequently occurs in recipients with NOD2 CD-associated SNPs. Intestinal crypt apoptosis, followed by extensive submucosal inflammation, might be provoked by microbes unconstrained by epithelial AMPs. In the absence of an antimicrobial shield, microbes could physically gain access to the apical surface of the enterocytes, cause cellular damage and provoke inflammation, as proposed to explain $\mathrm{CD}^{11}{ }^{30}{ }^{31}$ Recent studies have demonstrated that changes in the level of expression of Paneth cell defensins similar in magnitude to that observed in our transplant recipients can profoundly alter the numbers and species diversity of intestinal commensal bacteria. ${ }^{13}$ Alternatively, rejection might be a consequence of other compromised NOD2-dependent functions required for normal intestinal epithelial homeostasis. In either case, this process appears to differ with respect to the mechanism for "classical" tissue rejection, which is an inflammatory state provoked by antigens recognised as "non-self" by the recipient.

The allograft is depleted of donor lymphoid tissue prior to implantation; lymphoid cells of the recipient repopulate the allograft over the course of days. ${ }^{32}$ In contrast, the epithelial tissue of the allograft retains the donor genotype, with recipient epithelial cells comprising $<0.5 \%$, when examined between about 6 months and $>2$ years after the procedure. ${ }^{33}$ Hence, the NOD2-bearing cells of the recipient most probably contributing to allograft rejection and depressed AMP expression are the macrophages and/or dendritic cells that repopulate the allograft.

We can only speculate at this time on the mechanisms by which the macrophage and/or dendritic cell could influence the intestinal epithelium to modulate AMP expression. Recently published studies suggest that intestinal epithelial growth factors, such as Wnt, play a role in man in intestinal stem cell proliferation, cell fate determination and the physiological maintenance of mature Paneth cells. ${ }^{34-36}$ Indeed, in fully differentiated Paneth cells, induction of certain antimicrobial peptides and proteins, such as HD5 and HD6, appears to be actively regulated by Wnt. ${ }^{34-36}$ Precisely how signals from Wnt and other growth factors involved in the maintenance of a "healthy" intestinal epithelium are regulated to meet physiological needs is not known. Our study suggests that macrophages/dendritic cells, utilising NOD2-dependent circuits, might be helping to influence the outcome.

Holler et al have recently reported that transplantation of haematopoietic stem cells from donors with one or more CDassociated NOD2 polymorphisms increases the risk of severe intestinal graft versus host disease, a condition that resembles allograft rejection with respect to microscopic pathology. ${ }^{37} \mathrm{We}$ conclude that the risk of severe intestinal epithelial injury is increased when we repopulate the bowel with haematopoietic tissue bearing these NOD2 polymorphisms, regardless of whether we transplant the haematopoietic system or the intestine.

The high frequency of NOD2 polymorphisms discovered in our allograft recipient population is notable. Whereas the frequency of NOD2 CD-associated SNPs among our donors (previously healthy individuals with no gastrointestinal disease) was similar to that of the general population, the frequency of these polymorphisms among the recipients was similar to that reported for $\mathrm{CD}$ in various populations. ${ }^{4}$ The recipients are individuals who previously suffered one of several extreme intestinal insults requiring extensive surgical resection of the small intestine, or who suffered a disabling functional intestinal disorder. The high frequency of CD-associated NOD2 poly-

Table 2 Multivariate Cox proportional hazards analysis of pretransplantation factors affecting intestinal allograft loss

\begin{tabular}{lllllll}
\hline Variable & df & $\begin{array}{l}\text { Parameter } \\
\text { estimate }\end{array}$ & SE & $\chi^{2}$ & $\mathbf{p}>\chi^{2}$ & $\begin{array}{l}\text { Hazard ratio (95\% Cl for } \\
\text { hazard ratio) }\end{array}$ \\
\hline Recipient age & 1 & 0.03790 & 0.01976 & 3.6797 & 0.0551 & 1.039 (0.99 to 1.080) \\
Recipient CMV status & 1 & 3.14969 & 1.44126 & 4.7759 & 0.0289 & 0.043 (0.003 to 0.723) \\
Recipient NOD2 mutant 1 & 4.57855 & 1.66650 & 7.5482 & 0.0060 & 97.373 (3.715 to 2552.48) \\
\hline
\end{tabular}

A Cox proportional hazards model was used. df, degrees of freedom. 

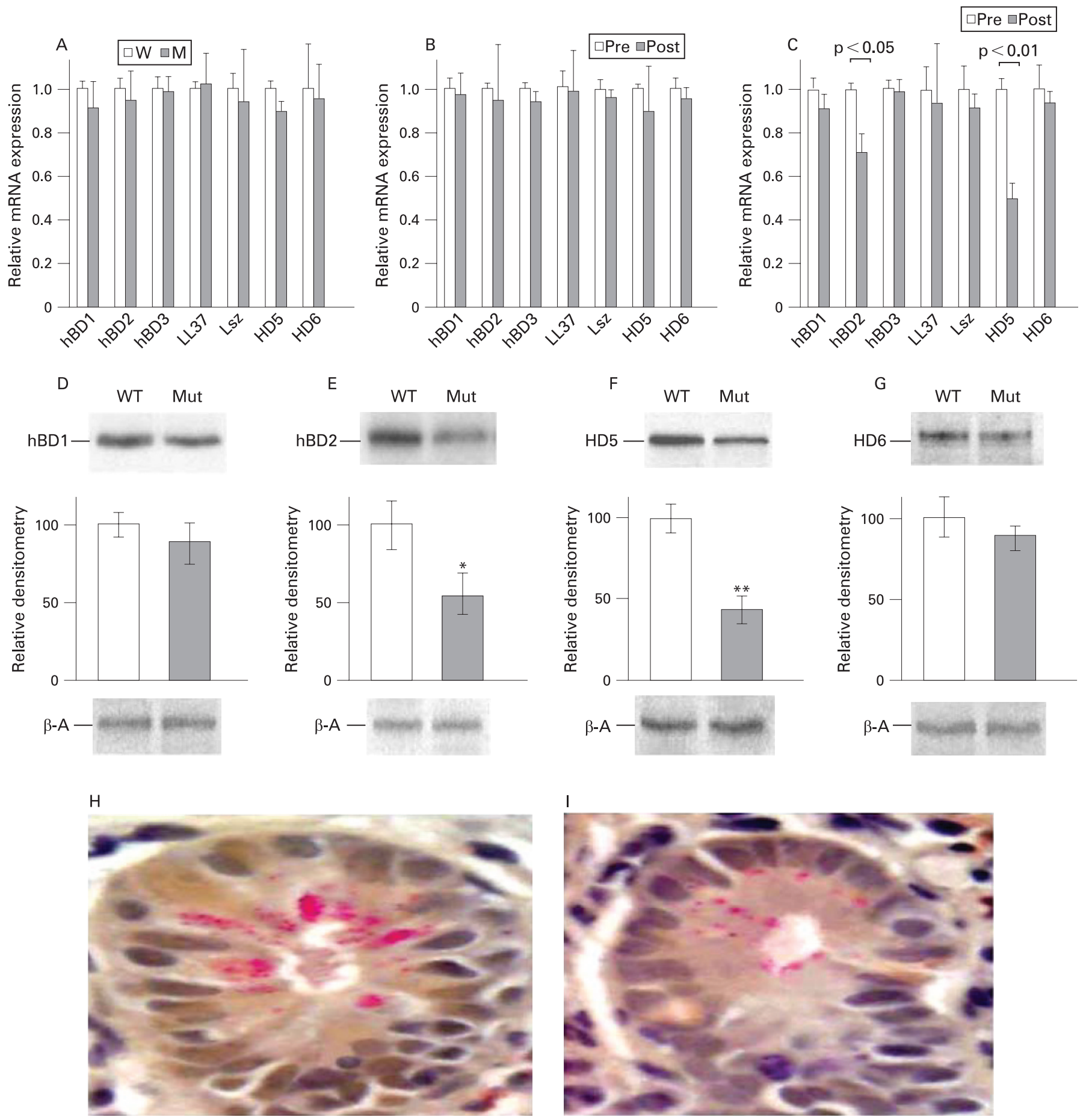

WT

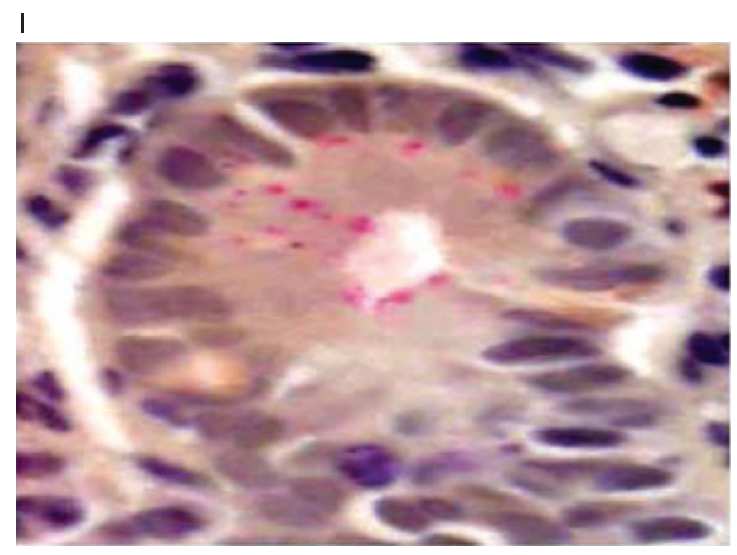

Mut

Figure 3 Expression of intestinal antimicrobial polypeptide (AMP) genes in tissue specimens from allografts in recipients with or without NOD2 Crohn's disease (CD)-associated polymorphisms. Recipients represent those in the transplant population that had not as yet experienced a rejection episode and had normal intestinal histology. All donor organs were from NOD2 wild-type donors (see text). Total RNA was isolated and analysed as described in the Methods section. The relative level (normalised to glyceraldehyde phosphate dehydrogenase) of target mRNA was determined by quantitative reverse transcriptase-PCR. Data are expressed as relative levels of each mRNA, as described in the figure, with the comparator assigned a value of 1.0. (A) Organs sampled prior to transplantation; tissue levels of specific AMPs from allografts implanted into recipients with a NOD2 wild-type genotype (W, white bars) $(N=6)$ are compared with those implanted into recipients with NOD2 mutant genotypes $(M$, grey bars) ( $N=4)$; $(B)$ AMP mRNA recovered from allografts, 3-4 weeks after transplantation into recipients with wild-type NOD2 loci; white bars, tissue samples taken from the donor bowel prior to transplantation; grey bars, biopsies taken from the same bowel 3-4 weeks after implantation. (C) as B, but allografts were transplanted into recipients with NOD2 CD-associated single nucleotide polymorphisms. (D-G) Western blot analyses of AMPs extracted from allograft biopsies taken at the same time points as in $B$ and $C$, and performed as described in the Methods section. Amounts of protein extract were normalised based on $\beta$-actin $(\beta-A)$ present. Quantitative densitometry of the photographic images for each peptide is presented, along with one representative blot image $\left({ }^{*} \mathrm{p}<0.05 ;{ }^{* *} \mathrm{p}<0.01\right)$. (H, I) Representative light microscopic images of Paneth cells taken from biopsy specimens corresponding to samples studied in $B$ and $C$, respectively (phloxine-tartrazine). 
morphisms observed in the recipient population might reflect a previously unappreciated genetic linkage with the specific intestinal disorders that ultimately required extensive surgical resection (eg, gastroschisis, malrotation, intestinal atresia, etc.). Alternatively, the presence of dysfunctional NOD2 alleles might impair the ability of the small intestine to accommodate the injurious "stresses" imposed by the primary intestinal disorder eventually leading to intestinal failure. Our study suggests that mutations in the NOD2 gene may be more widely associated with human gastrointestinal diseases than is currently appreciated.

In summary, NOD2 CD-associated polymorphisms in an intestinal allograft recipient appear to represent significant risk factors for rejection and death. An early event in the rejection process appears to involve impaired expression of epithelialderived AMPs, including those of the Paneth cell. Failure to protect the epithelium appears to be followed by visible damage to the epithelial barrier, and a subsequent inflammatory cell infiltration into the bowel wall. The association of this process with the NOD2 status of the recipient implicates a heretofore unrecognised role played by NOD2-expressing cells of bone marrow in the maintenance of normal intestinal epithelial immune health. Hopefully the information presented in this study will help guide our attempts to improve the outcome of small intestinal transplantation and other serious intestinal diseases in man.

Acknowledgements: Supported by grants from the Eli and Edyth L. Broad Medical Research Foundation (TF and MZ) and the American Society of Transplantation Surgeons-Wyeth Collaborative Scientist Research Award (TF and MZ). We wish to thank Dr Steve Evans (Surgery) for generous Departmental support and encouragement. We recognise Chad Owens for help in assembling clinical data. We are deeply grateful to the paediatric and adult nursing staff of the Georgetown University Hospital and to Erin Fennelly, RD for their tireless support and dedication to our patients.

Competing interests: None.

\section{REFERENCES}

1. Strober W, Murray PJ, Kitani A, et al. Signalling pathways and molecular interactions of NOD1 and NOD2. Nat Rev Immunol 2006;6:9-20.

2. Meylan E, Tschopp J, Karin M. Intracellular pattern recognition receptors in the host response. Nature 2006;442:39-44.

3. Ogura Y, Lala $\mathrm{S}$, Xin W, et al. Expression of NOD2 in Paneth cells: a possible link to Crohn's ileitis. Gut 2003;52:1591-7.

4. Cavanaugh J. NOD2: ethnic and geographic differences. World J Gastroenterol 2006;12:3673-7.

5. Hampe J, Cuthbert A, Croucher PJ, et al. Association between insertion mutation in NOD2 gene and Crohn's disease in German and British populations. Lancet 2001;357:1925-8.

6. Hampe J, Grebe J, Nikolaus S, et al. Association of NOD2 (CARD 15) genotype with clinical course of Crohn's disease: a cohort study. Lancet 2002;359:1661-5.

7. Ogura Y, Bonen DK, Inohara N, et al. A frameshift mutation in NOD2 associated with susceptibility to Crohn's disease. Nature 2001;411:603-6.

8. Hugot JP, Chamaillard M, Zouali $\mathrm{H}$, et al. Association of NOD2 leucine-rich repeat variants with susceptibility to Crohn's disease. Nature 2001;411:599-603.

9. Zasloff M. Antimicrobial peptides of multicellular organisms. Nature 2002;415:389-95.
10. Zasloff M. Antimicrobial peptides in health and disease. N Engl J Med 2002;347:1199-200.

11. Wehkamp J, Schmid M, Fellermann K, et al. Defensin deficiency, intestinal microbes, and the clinical phenotypes of Crohn's disease. J Leukoc Biol 2005;77:460-5

12. Kobayashi KS, Chamaillard M, Ogura Y, et al. Nod2-dependent regulation of innate and adaptive immunity in the intestinal tract. Science 2005;307:731-4.

13. Wehkamp J, Salzman NH, Porter E, et al. Reduced Paneth cell alpha-defensins in ileal Crohn's disease. Proc Natl Acad Sci USA 2005;102:18129-34.

14. Fishbein TM, Gondolesi GE, Kaufman SS. Intestinal transplantation for gut failure. Gastroenterology 2003;124:1615-28.

15. Reyes J, Todo S, Bueno J, et al. Intestinal transplantation in children: five-year experience. Transplant Proc 1996;28:2755-6.

16. Kato T, Gaynor JJ, Selvaggi G, et al. Intestinal transplantation in children: a summary of clinical outcomes and prognostic factors in 108 patients from a single center. J Gastrointest Surg 2005;9:75-89; discussion.

17. Kato T, Tzakis AG, Selvaggi G, et al. Intestinal and multivisceral transplantation in children. Ann Surg 2006;243:756-64; discussion 64-6.

18. Fishbein TM. The current state of intestinal transplantation. Transplantation 2004;78:175-8.

19. Fishbein TM, Kaufman SS, Florman SS, et al. Isolated intestinal transplantation: proof of clinical efficacy. Transplantation 2003;76:636-40.

20. Podolsky DK. Inflammatory bowel disease. N Engl J Med 2002;347:417-29

21. Shanahan F. Crohn's disease. Lancet 2002;359:62-9.

22. Selvaggi G, Kato T, Gaynor JJ, et al. Analysis of rejection episodes in over 100 pediatric intestinal transplant recipients. Transplant Proc 2006:38:1711-2.

23. Tzakis AG, Kato T, Nishida S, et al. The Miami experience with almost 100 multivisceral transplants. Transplant Proc 2006;38:1681-2.

24. Lee RG, Nakamura K, Tsamandas AC, et al. Pathology of human intestinal transplantation. Gastroenterology 1996;110:1820-34.

25. Ruiz $\mathbf{P}$, Bagni A, Brown $\mathrm{R}$, et al. Histological criteria for the identification of acute cellular rejection in human small bowel allografts: results of the pathology workshop at the VIII International Small Bowel Transplant Symposium. Transplant Proc 2004;36:335-7.

26. Ghosh D, Porter E, Shen B, et al. Paneth cell trypsin is the processing enzyme for human defensin-5. Nat Immunol 2002:3:583-90.

27. Buhner S, Buning C, Genschel J, et al. Genetic basis for increased intestinal permeability in families with Crohn's disease: role of CARD15 3020insC mutation? Gut 2006;55:342-7.

28. Wehkamp J, Stange EF. NOD2 mutation and mice: no Crohn's disease but many lessons to learn. Trends Mol Med 2005:11:307-9.

29. Wehkamp J, Harder J, Weichenthal M, et al. NOD2 (CARD15) mutations in Crohn's disease are associated with diminished mucosal alpha-defensin expression. Gut 2004:53:1658-64.

30. Swidsinski A, Loening-Baucke V, Theissig F, et al. Comparative study of the intestinal mucus barrier in normal and inflamed colon. Gut 2007:56:343-50.

31. Swidsinski A, Weber J, Loening-Baucke V, et al. Spatial organization and composition of the mucosal flora in patients with inflammatory bowel disease. J Clin Microbiol 2005;43:3380-9.

32. Iwaki Y, Starzl TE, Yagihashi A, et al. Replacement of donor lymphoid tissue in smallbowel transplants. Lancet 1991:337:818-9.

33. Tryphonopoulos $\mathbf{P}$, Kato T, Ruiz P, et al. Epithelial and hematopoietic cell chimerism in intestinal allografts. Transplant Proc 2004:36:359-60.

34. van Es JH, Jay $P$, Gregorieff $A$, et al. Wnt signalling induces maturation of Paneth cells in intestinal crypts. Nat Cell Biol 2005;7:381-6.

35. Gregorieff $\mathbf{A}$, Clevers $\mathrm{H}$. Wnt signaling in the intestinal epithelium: from endoderm to cancer. Genes Dev 2005;19:877-90.

36. Andreu $\mathbf{P}$, Colnot S, Godard C, et al. Crypt-restricted proliferation and commitment to the Paneth cell lineage following Apc loss in the mouse intestine. Development 2005; 132:1443-51.

37. Holler E, Rogler $\mathrm{G}$, Herfarth $\mathrm{H}$, et al. Both donor and recipient NOD2/CARD15 mutations associate with transplant-related mortality and GvHD following allogeneic stem cell transplantation. Blood 2004;104:889-94. 